\title{
O alendronato de sódio na prevenção da osteopenia secundária à imobilização gessada, em ratas: avaliação histomorfométrica*
}

\author{
Alendronate sodium to prevent osteopenia caused by cast \\ immobilization in female rats: histomorphometric evaluation
}

José B. Volpon ${ }^{1}$, Paulo Eugênio Santos Cecim², Cátia Isumi Miyase³, Nelson fabrício Gava ${ }^{4}$

\section{RESUMO}

Objetivo: Analisar, por meio da histomorfometria, o efeito do alendronato de sódio sobre o trabeculado ósseo de ratos, quando administrado simultaneamente com imobilização gessada. Métodos: Foram utilizados quatro grupos com cinco fêmeas de ratos Wistar: 1) imobilizado; 2) não imobilizado + alendronato; 3) imobilizado + alendronato; e 4) controle. A imobilização foi feita com gesso pelvipodálico aplicado até o membro posterior direito e o alendronato foi administrado em doses semanais. $O$ período de observação foi de $\mathbf{2 8}$ dias e realizada his-

* Trabalho realizado no Laboratório de Bioengenharia da Faculdade de Medicina da Universidade de São Paulo - USP - Ribeirão Preto (SP), Brasil.

1. Livre-docente, Professor Titular da Faculdade de Medicina de Ribeirão Preto da Universidade de São Paulo - USP - Ribeirão Preto (SP), Brasil.

2. Mestre, Médico do Departamento de Biomecânica Medicina e Reabilitação do Aparelho Locomotor da Faculdade de Medicina de Ribeirão Preto da Universidade de São Paulo - USP - Ribeirão Preto (SP), Brasil.

3. Residente de Ortopedia e Traumatologia do Hospital das Clínicas da Faculdade de Medicina de Ribeirão Preto da Universidade de São Paulo - USP - Ribeirão Preto (SP), Brasil.

4. Residente de Ortopedia e Traumatologia do Hospital das Clínicas da Faculdade de Medicina de Ribeirão Preto da Universidade de São Paulo - USP - Ribeirão Preto (SP), Brasil.

Endereço para correspondência: José B. Volpon, Rua São José, 655

- 14010-160 - Ribeirão Preto (SP), Brasil.

E-mail: jbvolpon@fmrp.usp.br

Recebido em 13/9/08. Aprovado para publicação em 8/10/08.

Copyright RBO2008 tomorfometria da metáfise proximal da tíbia, com análise do número de trabéculas, volume ósseo, espessura trabecular e separação trabecular. Resultados: O grupo imobilizado apresentou volume ósseo menor que os demais grupos. Os animais que receberam alendronato semanal, tanto imobilizados, quanto não imobilizados, apresentaram volume ósseo maior que o controle. A espessura trabecular no grupo imobilizado foi menor do que nos grupos controle e não imobilizado que recebeu alendronato, mas não apresentou diferença significativa em relação ao imobilizado com alendronato. $O$ grupo imobilizado apresentou separação trabecular maior que os demais grupos. Os grupos não imobilizado, sem imobilização que recebeu alendronato e imobilizado que recebeu alendronato apresentaram aumento no número de trabéculas em relação ao grupo imobilizado. Conclusão: A imobilização empregada efetivamente levou à osteopenia, verificada pela diminuição de todos os principais parâmetros histomorfométricos estudados. Estas alterações foram prevenidas pela administração concomitante de alendronato sódico, exceto com relação à espessura trabecular. $O$ alendronato de sódio foi capaz de aumentar os parâmetros morfométricos, mesmo em animais não imobilizados.

Descritores - Alendronato/administração \& dosagem; Osteoporose; Imobilização; Doenças ósseas metabólicas / prevenção \& controle; Microscopia; Ratos Wistar 


\section{ABSTRACT}

Objective: Using histomorphometric means to analyze the effect of alendronate sodium on the bone trabeculate of rats administered concomitantly with cast immobilization. Methods: Female Wistar rats were distributed in four groups with five animals each: 1) cast-immobilized; 2) no immobilization + alendronate; 3) cast-immobilized + alendronate; and 4) control. Immobilization was done with pelvipodalic cast applied till the right hind limb and alendronate was administered in weekly doses. The observation period was 28 days and histomorphometric evaluations were performed in the proximal tibial metaphysis, analyzing the number of trabeculae, bone volume, trabecular thickness, and trabecular separation. Results: The immobilized group presented less bone volume than the other groups. The animals receiving alendronate every week, whether or not immobilized, presented a greater bone volume than the control group. Trabecular thickness in the immobilized group was less than in the control and in the non-immobilized groups that received alendronate, but had no significant difference when compared to the immobilized with alendronate group. The immobilized group presented greater trabecular separation than the other groups. In the non-immobilized groups, the nonimmobilized group that received alendronate and the immobilized group that received alendronate presented an increased number of trabeculae when compared to the immobilized group. Conclusion: The immobilization used led to osteopenia, as confirmed by the decrease in all of the main histomorphometric parameters studied. Such changes were prevented with the concomitant administration of alendronate sodium, exception being made to the trabecular thickness. Alendronate sodium was able to increase morphometric parameters, even in non-immobilized animals.

Keywords - Alendronate/administration \& dosage; Osteoporosis; Immobilization; Bone diseases, metabolic/prevention \& control; Microscopy; Rats, Wistar

\section{INTRODUÇÃO}

O trofismo ósseo é dependente, entre outros fatores, das solicitações mecânicas que o esqueleto recebe. Em caso de diminuição da função, ou imobilização, o organismo parece reconhecer que nem toda a massa óssea é necessária para manter a integridade estrutural, o que leva a uma resposta que causa diminuição tanto da massa óssea, como da densidade mineral ${ }^{(1)}$.

No esqueleto eutrófico o osso está em contínua renovação e reabsorção. A situação clínica decorrente da alteração deste equilíbrio, por diminuição da neoformação e/ou por aumento da absorção, é chamada osteopenia ou, dependendo do grau, osteoporose. Na osteopenia humana há redução da densidade mineral óssea, entre 1,0 e 2,5 desvios-padrão abaixo da média da população adulta jovem, enquanto que na osteoporose a densidade mineral óssea está diminuída 2,5 ou mais desvios-padrão, associada à fratura com trauma pequeno $^{(2)}$. Estas situações podem ser decorrentes de alterações hormonais, síndromes, doenças osteometabólicas, ou situações que levam à imobilidade e redução da função, como nas sequielas neurológicas, período prolongado no leito ou por imobilizações. A imobilização estimula a reabsorção e deprime a formação óssea $^{(3)}$. Embora a ortopedia tenha evoluído no sentido de cada vez menos imobilizar o indivíduo e a fisioterapia esteja atuando precocemente, ainda um contingente apreciável de adultos e crianças fica com restrição da atividade física (hipoatividade) por períodos prolongados, em decorrência de morbidades de base.

Assim, quando a imobilização terapêutica for inevitável, buscam-se meios de atenuar ou neutralizar os efeitos adversos da hipoatividade por meio de vários métodos, dentre eles, o farmacológico. Muitos fármacos que atuam positivamente no metabolismo ósseo já foram testados com essa finalidade, sendo que um dos mais recentes é o alendronato de sódio ${ }^{(4-9)}$. Esta droga é da família dos bisfosfonatos que apresentam efeitos anti-reabsortivos bem documentados na literatura e a potência biológica depende de sua estrutura química individual $^{(1,10-14)}$. Como exemplos há o etiodronato, pamidronato, residronato, clodronato, alendronato, ibandronato e zolendronato ${ }^{(15-16)}$.

$\mathrm{O}$ alendronato de sódio, ou simplesmente alendronato, é um monossódio 4-amino-1-hidroxibutilidine bifosfonato que tem potência maior e grande seletividade para inibir a reabsorção óssea, sem afetar a for- 
mação óssea, o que não era observado com os outros compostos $^{(10-11,14-15,17) \text {. }}$

Hayes et al, em estudos em animais de laboratório, indicaram que, administrada sistemicamente essa droga é rapidamente levada ao tecido ósseo ou excretada pelos rins. Aproximadamente $60 \%$ a $70 \%$ da dose chega ao osso e a meia-vida é de cerca de 200 dias no rato e 100 dias no cão. Após sete semanas de administração de alendronato endovenoso aqueles autores observaram que ele é incorporado à matriz óssea e novo osso é formado em quantidade e qualidade normais ${ }^{(18)}$. Fleisch verificou que o alendronato não inibe a formação óssea e não atua no osteoclasto após incorporação na matriz óssea ${ }^{(1)}$.

A dose tóxica do alendronato foi estudada em animais. Por via oral, em ratos, é de aproximadamente $552 \mathrm{mg} / \mathrm{kg}\left(3.256 \mathrm{mg} / \mathrm{m}^{2}\right.$ equivalem à dose oral de humanos, $27.800 \mathrm{mg}$ ), em camundongos $966 \mathrm{mg} / \mathrm{kg}$ $\left(2.898 \mathrm{mg} / \mathrm{m}^{2}\right.$ equivalem à dose oral de humanos de $48.300 \mathrm{mg}$ ). A dose intravenosa foi 10 a 15 vezes menor que os valores da dose oral; entretanto, após a administração por essa via, os animais apresentaram primariamente alterações gastrintestinais e hemorra$\operatorname{gias}^{(16,18)}$.

Guy et al utilizaram alendronato administrado por via oral em ratos durante o crescimento, por 105 semanas. Os resultados comprovaram, pela radiografia da coluna lombar, a descalcificação da quarta vértebra lombar e pelo ensaio mecânico de compressão em três pontos do fêmur inteiro, que o alendronato administrado desde o crescimento inibiu a reabsorção óssea causando aumento da massa e atenuação da reabsorção durante o período da idade em que a perda óssea mais ocorreria. Mas, os autores descreveram importantes limitações. O estudo começou com machos e fêmeas de cinco semanas de idade, e as análises mostraram que o peso acumulado em machos foi aproximadamente $50 \%$ maior que nas fêmeas. Foi atribuída a este volume corporal a interferência nos resultados dos ensaios. Além disso, em função da idade dos animais para completar o estudo (105 semanas), ocorreu morte de aproximadamente $80 \%$ dos ratos por causas não relacionadas com o tratamento ${ }^{(17)}$.
Da Paz et al compararam os efeitos preventivos do alendronato com o do hormônio estrogênio sintetizado em ratas previamente ovariectomizadas. Concluíram que ambas as drogas foram efetivas, mas a dose de $0,1 \mathrm{mg} / \mathrm{kg} / \mathrm{dia}$ de alendronato forneceu melhores resultados $^{(19)}$.

Segundo Guarniero et al, os bisfosfonatos mais estudados como agentes que diminuem a reabsorção óssea ao atuar sobre os osteoclastos são o alendronato e o residronato, e podem ser indicados como terapia na osteoporose, tanto para mulheres, como para homens, e para o tratamento da osteoporose secundária induzida por corticosteróides ${ }^{(20)}$.

Até o presente, ainda não foi demonstrado claramente o efeito dos bisfosfonatos, particularmente do alendronato, na neutralização da osteopenia causada pela imobilização. Assim, o objetivo desta investigação foi analisar, por meio de estudos histomorfométricos, os efeitos do alendronato de sódio quando administrado preventivamente em situação que provoca osteopenia.

\section{MÉTODOS}

Este estudo foi conduzido de acordo com o Guide for the Care and Use of Laboratory Animals ${ }^{(21)} \mathrm{e}$ aprovado pela Comissão de Ética e Uso de Animais da Faculdade de Medicina de Ribeirão Preto, USP.

Foram utilizadas fêmeas de Rattus norvegicus albi$n u s$, variedade Wistar, com massa corporal entre $250 \mathrm{~g}$ e $300 \mathrm{~g}$, fornecidas pelo Biotério Central do Campus da USP em Ribeirão Preto. Todos os animais foram pesados no início do experimento e observados quanto ao estado geral e condições específicas das patas traseiras. As ratas foram divididas aleatoriamente em quatro grupos experimentais de cinco animais cada, no início do experimento. A caracterização de cada grupo está apresentada no quadro 1.

A imobilização utilizada foi por meio de aparelho gessado que incluiu a região lombar, pelve e o membro posterior direito, realizado segundo a técnica descrita por Carvalho et al ${ }^{(22)}$. Para a confecção do aparelho gessado, os animais foram anestesiados por via intraperitoneal com xylazina $20 \%$ (Rompun ${ }^{\circledR}$, Bayer) $12 \mathrm{mg} / \mathrm{kg}$ e ketamina $10 \%$ (Ketalar $^{\circledR}$, Parke-Davis) $95 \mathrm{mg} / \mathrm{kg}$, como descrito por outros autores ${ }^{(19,23)}$. 
QUADRO 1

Agrupamento e descrição dos procedimentos realizados

\begin{tabular}{|ccl|}
\hline \multicolumn{1}{|c|}{ Grupos } & \multicolumn{1}{c|}{ Descrição dos grupos } \\
\hline $\begin{array}{c}\text { Controle } \\
\text { (sem tratamento) }\end{array}$ & C & cinco ratas que permaneceram nas gaiolas por 28 dias. \\
\hline $\begin{array}{c}\text { Não imobilizadas } \\
+ \text { alendronato } \\
\text { semanal }\end{array}$ & ALN/S & $\begin{array}{l}\text { cinco ratas que permaneceram nas gaiolas e receberam } \\
\text { doses semanais }(0,7 \mathrm{mg} / \mathrm{kg}) \text { de alendronato por } 28 \text { dias. }\end{array}$ \\
\hline $\begin{array}{c}\text { Imobilizadas } \\
\text { Imobilizadas }+ \\
\text { alendronato } \\
\text { semanal }\end{array}$ & $\mathrm{I}+\mathrm{ALN} / \mathrm{S}$ & $\begin{array}{l}\text { cinco ratas que foram imobilizadas com aparelho ges- } \\
\text { sado pelvipodálico por } 28 \text { dias. }\end{array}$ \\
\hline $\begin{array}{l}\text { cinco ratas que foram imobilizadas com aparelho ges- } \\
\text { sado pelvipodálico e receberam doses semanais }(0,7 \mathrm{mg} / \\
\mathrm{kg}) \text { de alendronato por } 28 \text { dias. }\end{array}$ \\
\hline
\end{tabular}

Com o animal anestesiado, a região a ser imobilizada foi envolvida por malha tubular e ataduras de algodão. Na seqüência, foram utilizadas ataduras gessadas de $6,0 \mathrm{~cm}$ de largura, aplicadas de forma circular e uniforme, com o cuidado de não provocar compressão no membro ou causar áreas irregulares de apoio. $\mathrm{O}$ membro pélvico direito foi imobilizado com o quadril em extensão, discreta abdução e joelho em extensão. O aparelho gessado foi recortado de modo a deixar livre a região perineal, a pata direita e todo o membro pélvico esquerdo. As ratas foram inspecionadas diariamente para verificar possíveis complicações com a imobilização.

As ratas foram alojadas em número de dois em gaiolas comuns $(40 \times 33 \times 20 \mathrm{~cm})$, forradas com maravalhas e mantidas no biotério, com livre acesso à água e à alimentação (ração padrão), sob controle de temperatura $\left(23^{\circ} \mathrm{C} \pm 2^{\circ} \mathrm{C}\right)$ e luminosidade $(12 \times 12$ horas claro/escuro).

O alendronato foi manipulado da substância pura (alendronato sódico trihidratado 1\%) e acondicionado em frasco-ampolas de $10,0 \mathrm{~mL}$ na concentração de $10,0 \mathrm{mg} / \mathrm{mL}$, que é a máxima concentração em que se mantém solúvel. As doses utilizadas foram de $0,7 \mathrm{mg} /$ $\mathrm{kg}$ aplicadas subcutaneamente no dorso, uma vez por semana, durante $28 \operatorname{dias}^{(12,19)}$. Os animais foram mortos por meio de dose excessiva de tiopental sódico (Thionembutal ${ }^{\circledR}$ ) administrado intraperitonealmente. $\mathrm{O}$ aparelho gessado foi removido, e a tíbia do lado imo- bilizado foi desarticulada no joelho e no tornozelo e limpa das partes moles. Nos animais que não foram imobilizados, a tíbia direita foi desarticulada no joelho e no tornozelo e limpas das partes moles. Após inspeção macroscópica, os ossos foram envolvidos em gases umedecidas em solução de soro fisiológico e estocados em freezer, à temperatura de $-20^{\circ} \mathrm{C}$. Para as análises, os ossos foram retirados com antecedência de 12 horas e colocados em geladeira e, depois, à temperatura ambiente para descongelamento gradual.

\section{Preparo das lâminas histológicas}

As tíbias direitas, sem sofrerem descalcificação, foram processadas pela técnica descrita por Novaes Jr. et $a l^{(24)}$. O material foi fixado em solução de formaldeído $4 \%, \mathrm{pH} 7$, por 10 dias, em seguida submetido à solução de etanol com desidratação em concentrações crescentes, até $100 \%$, e aí mantido por três dias e, posteriormente, transferidas para solução com resina $L R$ White $^{\circledR}$ (London Resin Company), em agitação por 60 minutos e, na seqüência, mantidas em refrigeração no período noturno. No dia seguinte, a resina foi trocada e as peças colocadas em vácuo por 60 minutos, agitadas por mais uma hora, retornando ao refrigerador. Esse procedimento foi repetido por nove dias, com trocas da resina a cada 48 horas. No décimo dia, as peças foram incluídas em fôrmas (teflon) com nova resina e levadas à estufa $\left(60^{\circ} \mathrm{C}\right)$, durante a noite, para a polimerização. 

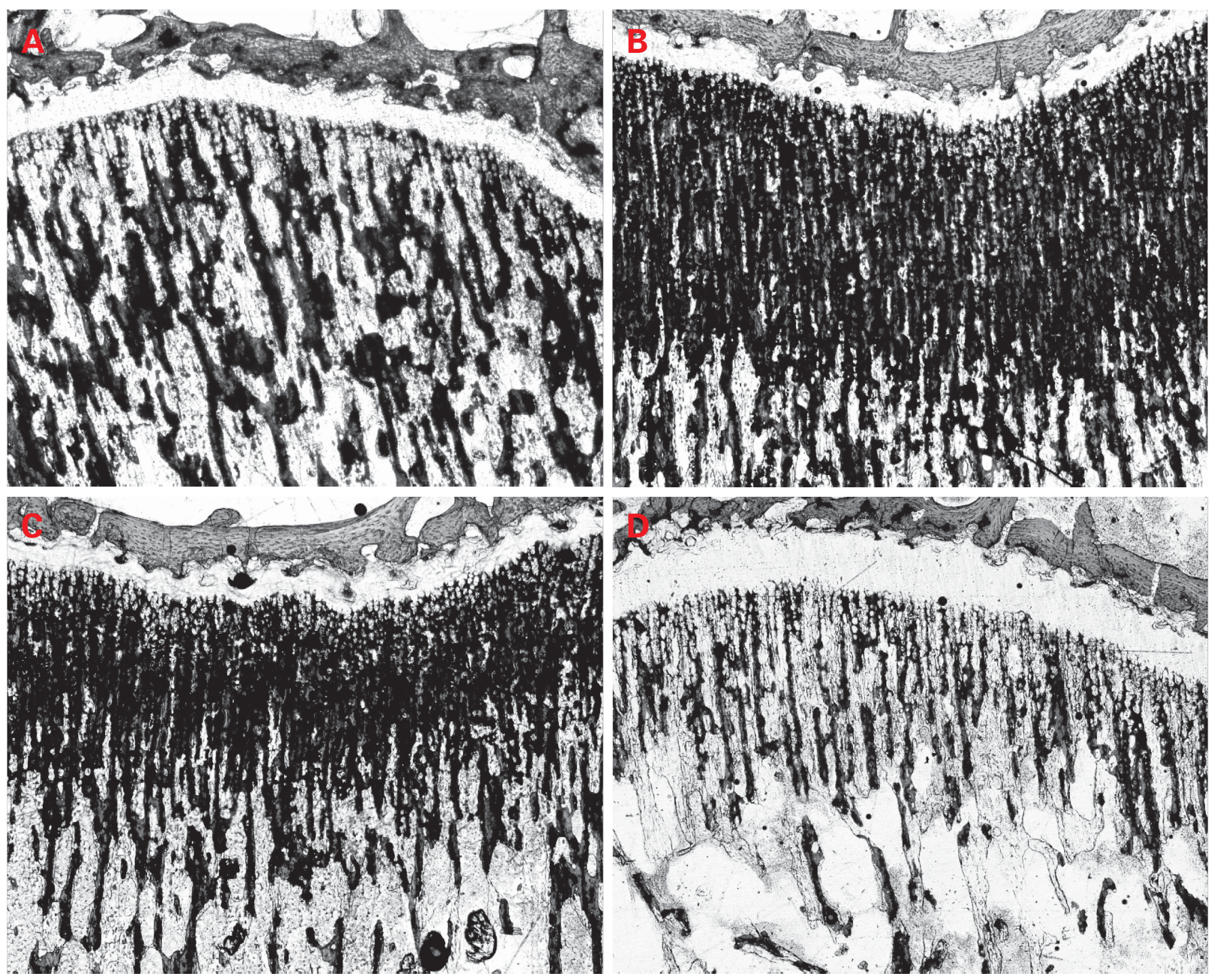

Figura 1 - Aspectos microscópicos, em corte frontal da metáfise proximal da tíbia, dos quatro grupos de animais. A) grupo controle; B) grupo não imobilizado que recebeu alendronato semanal; C) grupo imobilizado que recebeu alendronato; D) grupo apenas imobilizado. Percebe-se maior concentração de trabeculado nos grupos que receberam alendronato, mesmo com a imobilização. $\mathrm{O}$ grupo apenas imobilizado (D) mostra redução acentuada do trabeculado (coloração: azul de toluidina; aumento original: 20x).

O material, em blocos, foi secionado longitudinalmente por máquina de corte ISOMET $1000^{\circledR}$ (Buehler), em fatias com espessura de $100 \mu \mathrm{m}$, sob refrigeração com água, com velocidade programada e constante. As faces foram desgastadas e polidas e, então, o espécime colado à lâmina de vidro (Poxi-bonder). Os fragmentos ósseos colados foram novamente cortados entre 30 a $40 \mu \mathrm{m}$, desgastados até atingirem 10 a $15 \mu \mathrm{m}$ de espessura e corados em azul de toluidina.

\section{Análise histomorfométrica dos cortes} histológicos

Foi utilizado um microscópio Nikon Labophot-2 ${ }^{a \circledast}$ acoplado a um sistema semi-automático de histomorfometria Osteomeasure ${ }^{\circledR}$ (Osteometrics, Inc., Atlanta, GA, USA) e, com aumento de 20 vezes, foram analisa-

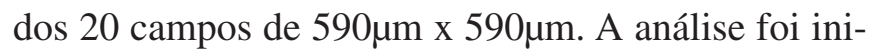
ciada no córtex, um campo abaixo da cartilagem de crescimento, até chegar ao córtex oposto, e, depois, 


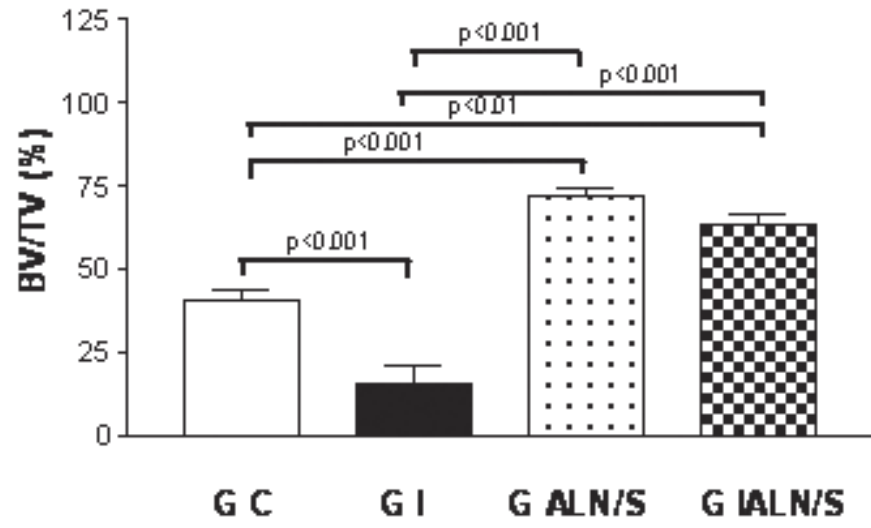

Gráfico 1 - Representação do volume ósseo encontrado nos quatro grupos. [GC (controle); GI (imobilizado); G ALN/S (não imobilizado + alendronato semanal); G IALN/S (imobilizado + alendronato semanal)].

continuar na fileira imediatamente abaixo no sentido contrário até englobar os 20 campos. Os parâmetros histomorfométricos estudados foram os estruturais, seguindo a nomenclatura proposta por Parfitt et al e padronizada pela American Society of Bone and Mineral Research, que são: o volume trabecular (BV/TV (\%)) que é o volume ocupado pelo osso trabecular, mineralizado ou não, expresso como percentagem do volume ocupado pela medula e trabéculas ósseas; espessura trabecular $(\mathrm{Tb} . \mathrm{Th}(\mu \mathrm{m}))$ que é a espessura das trabéculas ósseas; separação trabecular $(\mathrm{Tb} . \mathrm{Sp}(\mu \mathrm{m}))$ que é a distância entre os pontos médios das trabéculas ósseas; número de trabéculas $(\mathrm{Tb} . \mathrm{N}(/ \mathrm{mm}))$ que é o número de trabéculas ósseas por milímetro de teci$\mathrm{do}^{(25)}$.

\section{Análise estatística}

Foi utilizado o programa GraphPad Prism ${ }^{\circledR}$, versão 3.0 para Windows, 1999, onde foram empregados, para as variáveis numéricas que apresentaram normalidade, a ANOVA e o Teste de Comparação Múltipla de Tukey. O nível de significância adotado para rejeição da hipótese de nulidade foi de 5\%. Os resultados foram expressos como média e desvio-padrão $(\mathrm{x} \pm \mathrm{DP})$. A elaboração da apresentação gráfica dos dados foi realizada pelo programa GraphPad Prism, versão 3.0.

\section{RESULTADOS}

De maneira geral, à simples comparação visual das lâminas, já se podiam notar diferenças entre os gru-

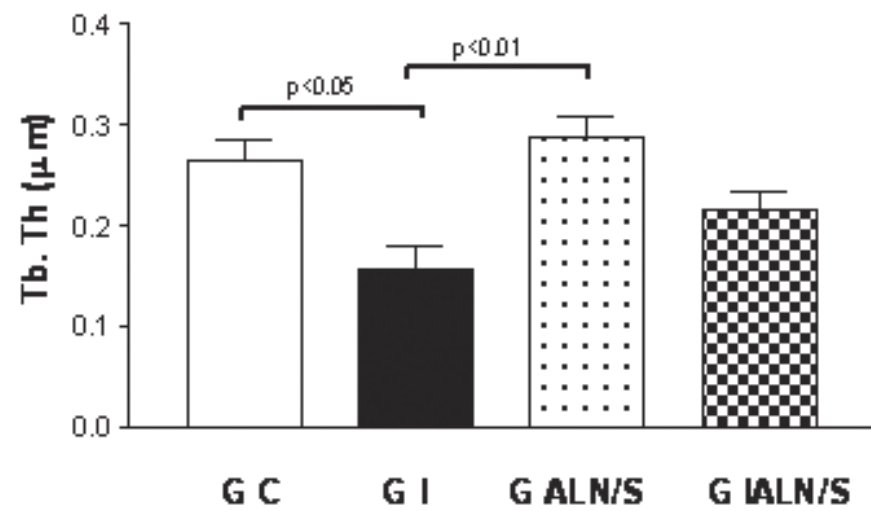

Gráfico 2 - Representação da espessura trabecular nos quatro grupos. [GC (controle); GI (imobilizado); G ALN/S (não imobilizado + alendronato semanal); G IALN/S (imobilizado + alendronato semanal)].

pos, pois nos animais que receberam alendronato, mesmos os imobilizados, havia maior concentração de trabeculado na região metafisária, enquanto que nos animais apenas imobilizados era patente a diminuição do trabeculado (figura 1).

$\mathrm{Na}$ avaliação do volume ósseo, o grupo imobilizado apresentou valores inferiores ao grupo controle e aos demais grupos $(\mathrm{p}<0,001)$, enquanto que os grupos que receberam alendronato semanal, tanto os imobilizados ( $\mathrm{p}<0,01$ ), como os não imobilizados apresentaram volume ósseo maior que o grupo controle $(\mathrm{p}<$ 0,001) (gráfico 1).

Com relação à espessura trabecular, no grupo imobilizado ela foi menor que no grupo controle $(p<0,05)$ e no grupo sem imobilização + alendronato semanal $(\mathrm{p}<0,01)$, mas não apresentou diferença significativa em relação ao grupo imobilizado + alendronato semanal. Não houve diferença significativa na espessura trabecular entre o grupo controle e os grupos que receberam alendronato (gráfico 2).

A separação trabecular no grupo imobilizado foi maior que nos demais grupos $(\mathrm{p}<0,01)$. Não houve diferença significativa na separação trabecular entre os outros grupos (gráfico 3 ).

O grupo não imobilizado + alendronato semanal apresentou aumento no número de trabéculas em relação ao grupo imobilizado $(\mathrm{p}<0,001)$ e ao grupo controle $(\mathrm{p}<0,05)$. $\mathrm{O}$ grupo imobilizado + alendronato semanal também apresentou aumento no número de 


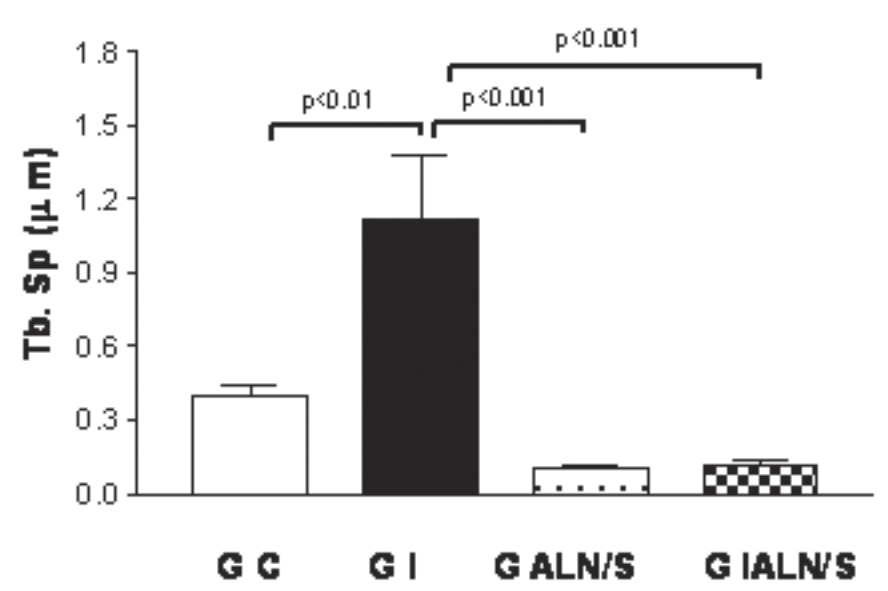

Gráfico 3 - Representação da separação trabecular nos quatro grupos. [GC (controle); GI (imobilizado); G ALN/S (não imobilizado + alendronato semanal); G IALN/S (imobilizado + alendronato semanal)].

trabéculas em relação ao grupo imobilizado $(\mathrm{p}<0,001)$ e ao grupo controle $(\mathrm{p}<0,001)$. Não houve diferença significativa no número de trabéculas entre o grupo controle e o grupo imobilizado (gráfico 4).

\section{DISCUSSÃO}

O equilíbrio entre a reabsorção e a formação de tecido ósseo é influenciado por vários fatores, como nutrição, atividade hormonal, atividade física (solicitação mecânica), e outros. Assim, a ingestão ou absorção inadequada de cálcio, afecções que causam alterações na produção e ou na atividade de hormônios (dentre eles paratormônio, insulina e calcitonina) podem levar ao desequilíbrio entre formação e reabsorção óssea, contribuindo, conseqüentemente, para o enfraquecimento do tecido ósseo.

Não menos importante é a solicitação mecânica que, quando diminuída por hábitos sedentários, pelo desuso causado por condições sistêmicas ou regionais como a imobilização, leva a um processo de adaptação com aumento da reabsorção óssea e, em decorrência, ao enfraquecimento ósseo caracterizando a osteopenia. $\mathrm{O}$ enfraquecimento do tecido ósseo é observado durante imobilizações terapêuticas que podem permanecer por períodos mais longos, com perturbação da homeostase cálcica ${ }^{(26)}$.

A prevenção da osteopenia secundária à hipoatividade pode ser realizada pela reabilitação que, entre-

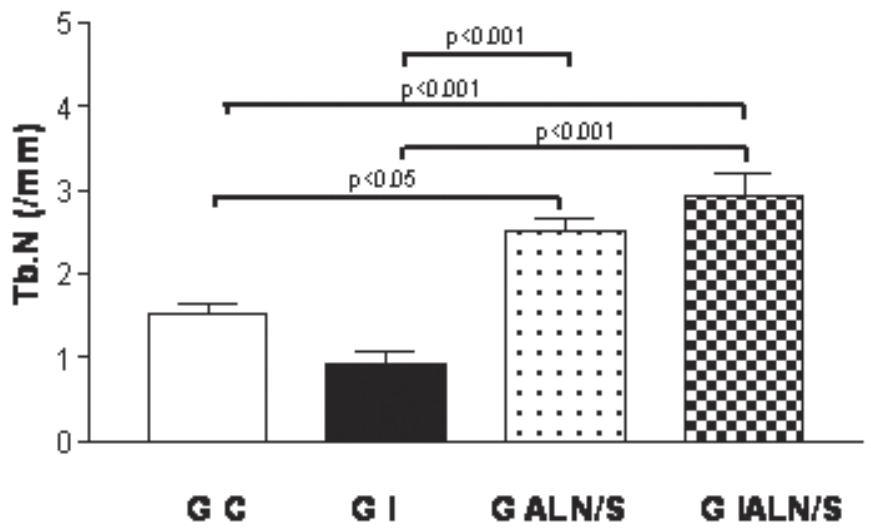

Gráfico 4 - Representação do número de trabéculas nos quatro grupos. GC (controle); GI (imobilizado); G ALN/S (não imobilizado + alendronato semanal); G IALN/S (imobilizado + alendronato semanal).

tanto, fica cerceada em caso de imobilizações extensas, donde o interesse na associação de substâncias que atuam positivamente no trofismo ósseo, como o cálcio $^{(27)}$, a calcitonina ${ }^{(28)}$ e os bisfosfonatos ${ }^{(7,10,29)}$.

Inicialmente usados para a prevenção e tratamento da osteoporose pós-menopausa ${ }^{(30)}$, os bisfosfonatos têm sido cada vez mais indicados para outras condições como osteogênese imperfeita, displasia fibrosa e osteoporose idiopática juvenil, além de ser utilizado nas metástases de câncer de mama, mieloma múltiplo e tumor gigantocelular ${ }^{(31)}$, bem como adjuvantes no tratamento da osteoporose encontrada em crianças com paralisia cerebral, betatalassemia e doença de Gaucher ${ }^{(13)}$. Autores descreveram os resultados de trabalhos com uso de bisfosfonatos em modelos animais na afecção de Legg-Calvé-Perthes visando, pela ação antiosteoclástica, atenuar a fase de reabsorção ${ }^{(32)}$.

Assim, os bisfosfonatos são um grupo de drogas potentes que atuam na inibição da atividade osteoclástica, onde quer que ela seja necessária ${ }^{(33)}$. Isto explica a variedade de suas indicações.

Poucos são os trabalhos que investigaram o uso do alendronato no combate à osteopenia secundária à imobilização prolongada. Mosekilde et al compararam o efeito do residronato e alendronato na prevenção de osteopenia em ratas imobilizadas $^{(8)}$. Leblanc et al simularam o efeito da ausência da gravidade em vôos espaciais e analisaram indivíduos normais que fica- 
ram confinados no leito(7). Em ambas as pesquisas, aqueles que receberam alendronato apresentaram melhores resultados densitométricos e laboratoriais de cálcio e eletrólitos.

Na presente investigação o objetivo foi avaliar se a administração do alendronato de sódio pode prevenir a osteopenia causada pela imobilização gessada em ratas, tendo como parâmetro a análise histomorfométrica. Por esta razão usamos os parâmetros propostos por Parfitt et al e recomendados pela American Society of Bone and Mineral Research (25).

A análise de nossos resultados mostra que a imobilização realizada efetivamente diminuiu a qualidade óssea, avaliada pela comparação dos parâmetros do trabeculado que se apresentou mais fino, menos denso e com maior espaçamento, no grupo imobilizado. Cecim usou o mesmo modelo de imobilização e, com testes mecânicos, comprovou a diminuição da resistência óssea após a imobilização( ${ }^{(29)}$. O modelo por nós adotado é adequado e corrobora os achados de outros autores no sentido de que o rato é animal indicado para este tipo de experimento, pelas características de resposta semelhante ao visto no osso humano ${ }^{(5,34-37)}$. A imobilização de ratos provoca perda de osso esponjoso caracterizada por falhas na formação óssea e aumento na reabsorção. A perda focal também ocorre em osso cortical, quando a imobilização for suficientemente prolongada, com concomitante diminuição nas propriedades mecânicas ${ }^{(5,38)}$.

Entretanto, como ponderado por Mosekilde et al, a extrapolação para humanos de dados obtidos em ratos deve ser vista com cautela porque os ratos são quadrúpedes e o sistema de remodelação óssea pode não mimetizar exatamente o que acontece no ser humano ${ }^{(8)}$.

Nossos dados indicam que o alendronato, utilizado na dose semanal de $0,7 \mathrm{mg} / \mathrm{kg}$ em ratas, foi eficaz para aumentar o volume ósseo e o número de trabéculas, mesmo nos animais imobilizados, o que significa uma alteração positiva na arquitetura e qualidade ósseas que levaram à neutralização dos efeitos da imobilização. Outro detalhe é que mesmo os animais não imobilizados que receberam tratamento apresentaram ossos mais densos.
Em acordo com nossos estudos estão os achados de Murakami et al que mostraram que o bisfosfato de tiludronato influencia a massa, estrutura e turnover do osso esponjoso ${ }^{(39-41)}$. Mosekilde et al chegaram a resultado semelhante, em relação ao risedronato e alendronato $^{(8)}$.

Esta pesquisa reforça outras que, em relação à imobilização, sugerem que seja recomendável encurtá-la ao máximo, ou restringi-la ao menor segmento possível $^{(7-8,26,29)}$. Neste aspecto, os dados experimentais dão suporte ao que já se faz em termos de tratamento tanto no campo da ortopedia, quanto no da fisioterapia.

Em condições de imobilização irreversíveis como as plegias conseqüentes às lesões espinhais ou cerebrais irreversíveis, o tratamento preventivo com bisfosfonatos seria recomendável, ainda com base em trabalhos experimentais ${ }^{(8)}$. Nestes casos deve-se tomar cuidado com o uso oral do medicamento pelos efeitos irritativos sobre o aparelho digestivo. Entretanto, mesmo em relação a este aspecto, há tendência de surgirem bisfosfonatos de efeito mais prolongado, quando aplicados por via endovenosa, como o ácido zoledrônico que em uma única dose anual pode ser um tratamento eficaz para osteoporose pós-menopausa ${ }^{(42-43)}$.

\section{CONCLUSÕES}

1) A imobilização empregada efetivamente levou à osteopenia, verificada pela diminuição dos principais parâmetros histomorfométricos estudados. Estas alterações foram prevenidas pela administração concomitante de alendronato sódico, exceto quanto à espessura trabecular.

2) O alendronato de sódio foi capaz de aumentar os parâmetros morfométricos, mesmo em animais normais.

\section{REFERÊNCIAS}

1. Fleisch H. Bisphosphonates in osteoporosis. Eur Spine J. 2003; 12 Suppl 2:S142-6.

2. Kanis JA, Melton LJ 3rd, Christiansen C, Johnston CC, Khaltaev N. The diagnosis of osteoporosis. J Bone Miner Res. 1994;9(8):1137-41. Comment in: J Bone Miner Res. 1996; 11(8):1199-201.

3. Cavanagh PR, Licata AA, Rice AJ. Exercise and pharmacological countermeasures for bone loss during long- 
duration space flight. Gravit Space Biol Bull. 2005;18(2):3958.

4. Bikle DD, Sakata T, Halloran BP. The impact of skeletal unloading on bone formation. Gravit Space Biol Bull. 2003; 16(2):45-54. Review.

5. Egermann M, Goldhahn J, Schneider E. Animal models for fracture treatment in osteoporosis. Osteoporos Int. 2005;16 Suppl 2:S129-38.

6. Ke HZ, Crawford DT, Qi H, Simmons HA, Owen TA, Paralkar $\mathrm{VM}$, et al. A nonprostanoid EP4 receptor selective prostaglandin E2 agonist restores bone mass and strength in aged, ovariectomized rats. J Bone Miner Res. 2006;21(4):56575.

7. LeBlanc AD, Driscol TB, Shackelford LC, Evans HJ, Rianon NJ, Smith SM, et al. Alendronate as an effective countermeasure to disuse induced bone loss. J Musculoskelet Neuronal Interact. 2002;2(4):335-43.

8. Mosekilde L, Thomsen JS, Mackey MS, Phipps RJ. Treatment with risedronate or alendronate prevents hind-limb immobilization-induced loss of bone density and strength in adult female rats. Bone. 2000;27(5):639-45.

9. Oliveira LAA, Guarniero R, Rodrigues CJ, Santana PJ, Batista MA. Avaliação do efeito do Risedronato Sódico na consolidação de fraturas: estudo experimental em ratos. Acta Ortop Bras. 2004;12(2):77-83.

10. Morris CD, Einhorn TA. Bisphosphonates in orthopaedic surgery. J Bone Joint Surg Am. 2005;87(7):1609-18.

11. Porras AG, Holland SD, Gertz BJ. Pharmacokinetics of alendronate. Clin Pharmacokinet. 1999;36(5):315-28.

12. Seedor JG, Quartuccio HA, Thompson DD. The bisphosphonate alendronate (MK-217) inhibits bone loss due to ovariectomy in rats. J Bone Miner Res. 1991;6(4):339-46.

13. Srivastava T, Alon US. The role of bisphosphonates in diseases of childhood. Eur J Pediatr. 2003;162(11):735-51.

14. Szejnfeld VL. Osteoporose: diagnóstico e tratamento. São Paulo: Sarvier; 2000. p. 353-66.

15. Marcus R. Fármacos que afetam a calcificação e a renovação óssea. In: Goodman LS, Gilman AG, Hardman JG, Limbird LE. Goodman \& Gilman: as bases farmacológicas da terapêutica. 10a. ed. Rio de Janeiro: McGraw-Hill; 2003. p. 1291-311.

16. Peter CP, Kindt MV, Majka JA. Comparative study of potential for bisphosphonates to damage gastric mucosa of rats. Dig Dis Sci. 1998;43(5):1009-15. Comment in: Dig Dis Sci. 1999; 44(4):728. Dig Dis Sci. 1999;44(2):311-3.

17. Guy JA, Shea M, Peter CP, Morrissey R, Hayes WC. Continuous alendronate treatment throughout growth, maturation, and aging in the rat results in increases in bone mass and mechanical properties. Calcif Tissue Int. 1993;53(4): 283-8.

18. Hayes WC, Shea M, Rodan GA. Preclinical evidence of normal bone with alendronate. Int J Clin Pract Suppl. 1999;101:9-13.
19. da Paz LH, de Falco V, Teng NC, dos Reis LM, Pereira RM, Jorgetti V. Effect of 17 beta-estradiol or alendronate on the bone densitometry, bone histomorphometry and bone metabolism of ovariectomized rats. Braz J Med Biol Res. 2001; 34(8):101522.

20. Guarniero R, Oliveira LG. Osteoporose: atualização no diagnóstico e princípios básicos para o tratamento. Rev Bras Ortop. 2004;39(9):477-85.

21. Institute of Laboratory Animal Resources (U.S.). Guide for the care and use of laboratory animals. 7th ed. Washington, D.C.: National Academy Press; 1996.

22. Carvalho CMM, Shimano AC, Volpon JB. Efeitos da imobilização e do exercício físico em algumas propriedades mecânicas do músculo esquelético. Rev Bras Eng Biom = Braz J Biomed Eng. 2002;18(2):65-73.

23. Coutinho EL, Gomes AR, França CN, Salvini TF. A new model for the immobilization of the rat hind limb. Braz J Med Biol Res. 2002;35(11):1329-32.

24. Novaes AB Jr, de Oliveira RR, Taba Júnior M, de Souza SL, Palioto DB, Grisi MF, Papalexiou V. Crestal bone loss minimized when following the crestal preparation protocol: a histomorphometric study in dogs. J Oral Implantol. 2005; 31(6):276-82.

25. Parfitt AM, Drezner MK, Glorieux FH, Kanis JA, Malluche H, Meunier PJ, et al. Bone histomorphometry: standardization of nomenclature, symbols, and units. Report of the ASBMR Histomorphometry Nomenclature Committee. J Bone Miner Res. 1987;2(6):595-610.

26. Akkari M, Santili C. Metabolismo do cálcio e do fósforo. In: Pardini Júnior AG, Souza JMG, Cunha LAM, editores. Clínica ortopédica. Rio de Janeiro: Medsi; 2003. p. 253-9.

27. Ke HZ, Qi H, Crawford DT, Simmons HA, Xu G, Li M, et al. A new vitamin $\mathrm{D}$ analog, $2 \mathrm{MD}$, restores trabecular and cortical bone mass and strength in ovariectomized rats with established osteopenia. J Bone Miner Res. 2005;20(10):1742-55.

28. Jiang Y, Zhao J, Geusens P, Liao EY, Adriaensens P, Gelan J, et al. Femoral neck trabecular microstructure in ovariectomized ewes treated with calcitonin: MRI microscopic evaluation. J Bone Miner Res. 2005;20(1):125-30.

29. Cecim PES. Uso de alendronato de sódio em ratas submetidas à imobilização gessada. Análise de propriedades mecânicas do fêmur e da densidade óssea radiográfica do colo femoral [dissertação]. São Paulo: Faculdade de Medicina de Ribeirão Preto da Universidade de São Paulo; 2007.

30. Liberman UA, Weiss SR, Bröll J, Minne HW, Quan H, Bell NH, et al. Effect of oral alendronate on bone mineral density and the incidence of fractures in postmenopausal osteoporosis. The Alendronate Phase III Osteoporosis Treatment Study Group. N Engl J Med. 1995;333(22):1437-43. Comment in: ACP J Club. 1996;124(2):33. N Engl J Med. 1995;333(22): 1495-6. N Engl J Med. 1996;334(11):733-4; author reply 734-5. N Engl J Med. 1996;334(11):734; author reply 734-5. N Engl J Med. 2001;344(20):1553-4; author reply 1554-6. 
31. Batch JA, Couper JJ, Rodda C, Cowell CT, Zacharin M. Use of bisphosphonate therapy for osteoporosis in childhood and adolescence. J Paediatr Child Health. 2003;39(2):88-92.

32. Little DG, McDonald M, Sharpe IT, Peat R, Williams P, McEvoy T. Zoledronic acid improves femoral head sphericity in a rat model of perthes disease. J Orthop Res. 2005;23(4): 862-8.

33. Reszka AA, Rodan GA. Bisphosphonate mechanism of action. Curr Rheumatol Rep. 2003;5(1):65-74.

34. Józsa L, Réffy A, Järvinen M, Kannus P, Lehto M, Kvist M. Cortical and trabecular osteopenia after immobilization. A quantitative histological study of the rat knee. Int Orthop. 1988; 12(2):169-72.

35. Brighton CT, Katz MJ, Goll SR, Nichols CE 3rd, Pollack SR. Prevention and treatment of sciatic denervation disuse osteoporosis in the rat tibia with capacitively coupled electrical stimulation. Bone. 1985;6(2):87-97.

36. Hott M, Deloffre P, Tsouderos Y, Marie PJ. S12911-2 reduces bone loss induced by short-term immobilization in rats. Bone. 2003;33(1):115-23.

37. Mosekilde L. Assessing bone quality-animal models in preclinical osteoporosis research. Bone. 1995;17(4 Suppl): 343S-352S.

38. Miller SC, Bowman BM, Jee WS. Available animal models of osteopenia - small and large. Bone. 1995;17(4 Suppl):117S$123 \mathrm{~S}$.

39. Murakami H, Nakamura T, Tsurukami H, Abe M, Barbier A, Suzuki K. Effects of tiludronate on bone mass, structure, and turnover at the epiphyseal, primary, and secondary spongiosa in the proximal tibia of growing rats after sciatic neurectomy. J Bone Miner Res. 1994;9(9):1355-64.

40. Murakami H, Takahashi N, Sasaki T, Udagawa N, Tanaka S, Nakamura I, et al. A possible mechanism of the specific action of bisphosphonates on osteoclasts: tiludronate preferentially affects polarized osteoclasts having ruffled borders. Bone. 1995;17(2):137-44.

41. Murakami H, Takahashi N, Tanaka S, Nakamura I, Udagawa N, Nakajo S, et al. Tiludronate inhibits protein tyrosine phosphatase activity in osteoclasts. Bone. 1997;20(5):399-404.

42. Black DM, Delmas PD, Eastell R, Reid IR, Boonen S, Cauley JA, Cosman F, Lakatos P, Leung PC, Man Z, Mautalen C, Mesenbrink P, Hu H, Caminis J, Tong K, Rosario-Jansen T, Krasnow J, Hue TF, Sellmeyer D, Eriksen EF, Cummings SR; HORIZON Pivotal Fracture Trial. Once-yearly zoledronic acid for treatment of postmenopausal osteoporosis. N Engl J Med. 2007;356(18):1809-22. Comment in: ACP J Club. 2007; 147(2):31. Curr Rheumatol Rep. 2008;10(2):89-91. Evid Based Med. 2007;12(5):145. Expert Opin Pharmacother. 2008; 9(4):663-8. N Engl J Med. 2007;357(7):711-2; author reply 714-5. N Engl J Med. 2007;357(7):711; author reply 714-5. N Engl J Med. 2007;357(7):712-3; author reply 714-5. N Engl J Med. 2007;357(7):713-4; author reply 714-5. N Engl J Med. 2007;357(7):713; author reply 714-5. N Engl J Med. 2007; 356(18):1878-80. N Engl J Med. 2007;356(18):1895-6. N Engl J Med. 2008;358(9):967-8; author reply 968-9. N Engl J Med. 2008;358(9):969; author reply 968-9.

43. McClung M, Recker R, Miller P, Fiske D, Minkoff J, Kriegman $\mathrm{A}$, et al. Intravenous zoledronic acid $5 \mathrm{mg}$ in the treatment of postmenopausal women with low bone density previously treated with alendronate. Bone. 2007;41(1):122-8. Comment in: Nat Clin Pract Endocrinol Metab. 2008;4(1):20-1. 